Ann. Funct. Anal. 6 (2015), no. 1, 34-53

http://doi.org/10.15352/afa/06-1-4

ISSN: 2008-8752 (electronic)

http://projecteuclid.org/afa

\title{
SOLUTIONS OF NONLINEAR ELLIPTIC PROBLEMS WITH LOWER ORDER TERMS
}

\author{
YOUSSEF AKDIM ${ }^{1}$, ABDELMOUJIB BENKIRANE ${ }^{2}$ AND MOSTAFA EL MOUMNI²* \\ Communicated by C. Cuevas
}

\begin{abstract}
We give an existence result for strongly nonlinear elliptic equations of the form

$$
-\operatorname{div}(a(x, u, \nabla u))+g(x, u, \nabla u)+H(x, \nabla u)=\mu \text { in } \Omega,
$$

where the right hand side belongs to $L^{1}(\Omega)+W^{-1, p^{\prime}}(\Omega)$ and $-\operatorname{div}(a(x, u, \nabla u))$ is a Leray-Lions type operator with growth $|\nabla u|^{p-1}$ in $\nabla u$. The critical growth condition on $g$ is with respect to $\nabla u$ and no growth condition with respect to $u$, while the function $H(x, \nabla u)$ grows as $|\nabla u|^{p-1}$.
\end{abstract}

\section{INTRODUCTION AND PRELIMINARIES}

In the present paper, we study the existence result for a class of nonlinear elliptic equations. The model problem is the following

$$
\begin{cases}-\nabla(A(x) \nabla u)+B(x, u, \nabla u)=\mu & \text { in } \Omega \\ u=0 & \text { in } \partial \Omega\end{cases}
$$

where $\Omega$ is a regular open bounded set in $\mathbb{R}^{N}(\mathrm{~N} \geq 1)$ and $B$ involves the unknown $u$ and its first derivatives. Precisely, $B$ splits into terms which are linear with respect to $u$ and $\nabla u$ and a nonlinear term as follows

$$
B(x, u, \nabla u)=b(x) \nabla u+g(x, u, \nabla u) .
$$

Here, $A$ and $b$ are given functions defined on $\Omega$ with values in $\mathbb{R}^{N} \times \mathbb{R}^{N}$ and $\mathbb{R}^{N}$, respectively. In fact, we focus our attention on the following problem

$$
\begin{cases}-\operatorname{div}(a(x, u, \nabla u))+g(x, u, \nabla u)+H(x, \nabla u)=f-\operatorname{div} F & \text { in } \Omega, \\ u=0 & \text { in } \partial \Omega\end{cases}
$$

Date: Received: Jan. 5, 2013; Revised: 24 Apr. 2013; Accepted: May 1, 2013.

* Corresponding author.

2010 Mathematics Subject Classification. Primary 35B45; Secondary 46E35, 35J60.

Key words and phrases. Sobolev spaces, a priori estimates, nonlinear elliptic equation. 
where $-\operatorname{div}(a(x, u, \nabla u))$ is a Leray-Lions operator acting from $W_{0}^{1, p}(\Omega)$ into its dual $W^{-1, p^{\prime}}(\Omega)$, which is coercive and grows like $|\nabla u|^{p-1}$ with respect to $\nabla u, p^{\prime}:=\frac{p}{p-1}$. Furthermore, The functions $g$ and $H$ are two the Carathéodory functions with suitable assumptions (see Assumption $\mathrm{H}(2)$ ). Main difficulties in this work arise from the fact that we consider data which only belong to $L^{1}(\Omega)+W^{-1, p^{\prime}}(\Omega)$, namely

$$
f \in L^{1}(\Omega) \text { and } F \in\left(L^{p^{\prime}}(\Omega)\right)^{N} .
$$

Many physicals models lead to elliptic and parabolic problems. For instance, in [17] the authors study the modeling of an electronic device. The derived elliptic system coupled the temperature (denoted $u$ ) and the electronically potential ( denoted $\Phi$ ). The temperature equation is considered as an elliptic equation where the second member $f=|\nabla \Phi|^{2}$ belongs to $L^{1}(\Omega)$. In [18] a Fokker-Planck equation arising in populations dynamics is studied. Models of turbulent flows in oceanography and climatology also lead to such kind of problems ( see [19] and the references therein).

In [20] the author studies the Navier-Stokes equations completed by an equation for the temperature $(u=T)$. In this case, if we denote by $v$ the velocity of the fluid, then the temperature equation reduces associate to (1.1). Note that for compressible flows the divergence of the velocity does not vanish, and the temperature equation can be considered with linear terms having the form $b(x) \nabla u$. These linear terms introduce new difficulties in the sense that the compactness results, do not apply directly to (1.1) which needs further technical investigations.

We are interested in existence results for weak solutions to (3.2). We have proved such an existence result, when $H \equiv 0$, there is a wide literature in which one can find existence results for problem (3.2). For instance, in the variational case (i.e. when $f \in W^{-1, p^{\prime}}(\Omega)$ ), existence result can be found in [10] while if $f \in L^{1}(\Omega)$ initiated basic works were given in $[15,12,25]$, also an existence result for (3.2) was proved in [11] (see the references therein). Related topics can be found in [24].

When $H$ is not necessarily the null function, existence result for problem (3.2) was proved first in [16] in the case where g does not depend on the gradient and then in [23] using, in both works, the rearrangement techniques. for different approach used in the setting of Orlicz Sobolev space the reader can refer to [3]-[8]. See also [9] for related topics

The main features of (3.2) are both the fact that the operator has two lower order terms, which produce a lack of coercivity and the right-hand side which is a measure. The operator has no lower order terms (i.e. $H \equiv g \equiv 0$ ), in this case the difficulties in studying problem (3.2) are due only to the right-hand side belong to $L^{1}(\Omega)+W^{-1, p^{\prime}}(\Omega)$. Simple examples (the Laplace operator in a ball, i.e. $p=2, H \equiv g \equiv 0$, and second member the Dirac mass in the center) show that, in general, the solution of (3.2) does not belong to the space $W_{l o c}^{1,1}(\Omega)$. Thus it is necessary to change the classical framework of Sobolev spaces in order to prove existence results. In the present paper we consider operators where both 
the lower order terms $H(x, \nabla u)$ appear without any coerciveness assumption on the operator.

Our aim in this paper is to investigate the existence of unbounded solutions to some the strongly nonlinear elliptic equations (3.2), in the case where the right-hand side belongs to $L^{1}(\Omega)+W^{-1, p^{\prime}}(\Omega)$. The function $g(x, s, \xi)$ is assumed to have exactly the natural growth (i.e. of order $p$ ), but no growth assumption is imposed with respect to s to the function $g$ which only satisfies the sign condition and the coercivity condition. The function $H(x, \xi)$, which induces a convection term, is assumed only to grow at most as $|\xi|^{p-1}$.

Now we state the Lemma is a slight modification of Gronwall's lemma (see [2]).

Lemma 1.1. Given the function $\lambda, \gamma, \varphi, \rho$ defined on $[a,+\infty[$, suppose that $a \geq 0$, $\lambda \geq 0, \gamma \geq 0$ and that $\lambda \gamma, \lambda \varphi$ and $\lambda \rho$ belong to $L^{1}(a,+\infty)$. If for a.e. $t \geq 0$ we have

$$
\varphi(t) \leq \rho(t)+\gamma(t) \int_{t}^{+\infty} \lambda(\tau) \varphi(\tau) d \tau
$$

then for a.e. $t \geq 0$

$$
\varphi(t) \leq \rho(t)+\gamma(t) \int_{t}^{+\infty} \rho(\tau) \lambda(\tau)\left(\int_{t}^{\tau} \lambda(r) \gamma(r) d r\right) d \tau
$$

We recall that, for $k>1$ and $s$ in $\mathbb{R}$, the truncation is defined as

$$
T_{k}(s)= \begin{cases}s & \text { if }|s| \leq k \\ k \frac{s}{|s|} & \text { if }|s|>k\end{cases}
$$

\section{MAin Results}

Let us now give the precise hypotheses on the problem (3.2), we assume that the following assumptions:

Assumption $\mathbf{H}(\mathbf{1}) . \Omega$ is a bounded open set of $\mathbb{R}^{N}(N \geq 1)$, Let $a: \Omega \times \mathbb{R} \times$ $\mathbb{R}^{N} \rightarrow \mathbb{R}^{N}$ be a Carathéodory function, such that

$$
|a(x, s, \xi)| \leq \beta\left[k(x)+|s|^{p-1}+|\xi|^{p-1}\right]
$$

for a.e. $(x) \in \Omega$, all $(s, \xi) \in \mathbb{R} \times \mathbb{R}^{N}$, some positive function $k(x) \in L^{p^{\prime}}(\Omega)$ and $\beta>0$.

$$
\begin{gathered}
{[a(x, s, \xi)-a(x, s, \eta)](\xi-\eta)>0 \text { for all }(\xi, \eta) \in \mathbb{R}^{N} \times \mathbb{R}^{N}, \text { with } \xi \neq \eta,} \\
\qquad a(x, s, \xi) \xi \geq \alpha|\xi|^{p}
\end{gathered}
$$

where $\alpha$ is a strictly positive constant. 
Assumption H(2). Furthermore, let $g(x, s, \xi): \Omega \times \mathbb{R} \times \mathbb{R}^{N} \rightarrow \mathbb{R}$ and $H(x, \xi)$ : $\Omega \times \mathbb{R}^{N} \rightarrow \mathbb{R}$ are two Carathéodory functions which satisfy, for almost every $(x) \in \Omega$ and for all $s \in \mathbb{R}, \xi \in \mathbb{R}^{N}$, the following conditions

$$
\begin{gathered}
|g(x, s, \xi)| \leq L_{1}(|s|)\left(L_{2}(x)+|\xi|^{p}\right), \\
g(x, s, \xi) s \geq 0,
\end{gathered}
$$

where $L_{1}: \mathbb{R}^{+} \rightarrow \mathbb{R}^{+}$is a continuous increasing function, while $L_{2}(x)$ is positive and belongs to $L^{1}(\Omega)$.

$$
\begin{gathered}
\exists \delta>0, \quad \nu^{\prime}>0: \text { for }|s| \geq \delta, \quad|g(x, s, \xi)| \geq \nu^{\prime}|\xi|^{p} \\
|H(x, \xi)| \leq b(x)|\xi|^{p-1}
\end{gathered}
$$

where $b(x)$ is positive and belongs to $L^{r}(\Omega)$ with $r>\max (N, p)$.

Assumption H(3). As far as the right-hand side of (3.2) is concerned, we assume that

$$
f \in L^{1}(\Omega) \text { and } F \in\left(L^{p^{\prime}}(\Omega)\right)^{N} .
$$

We shall use the following definitions of weak solutions for problem (3.2) in the following sense:

Definition 2.1. A weak solution of (3.2) is a measurable function $u: \Omega \rightarrow \overline{\mathbb{R}}$, such that

$$
\left\{\begin{array}{c}
u \in W_{0}^{1, p}(\Omega) \\
\int_{\Omega} a(x, u, \nabla u) \cdot \nabla v d x+\int_{\Omega} g(x, u, \nabla u) v d x+\int_{\Omega} H(x, \nabla u) v d x \\
=\int_{\Omega} f v d x+\int_{\Omega} F \cdot \nabla v d x \\
\forall \quad v \in W_{0}^{1, p}(\Omega) \cap L^{\infty}(\Omega)
\end{array}\right.
$$

Remark 2.2. Observe that, in (2.9), we can not replace $W_{0}^{1, p}(\Omega) \cap L^{\infty}(\Omega)$ by only $W_{0}^{1, p}(\Omega)$, since in general the two integrals $\int_{\Omega} g(x, u, \nabla u) v d x$ and $\int_{\Omega} f v d x$ may have no meaning.

We are interested in finding weak solutions of problem (3.2), i.e. solutions of (3.2) in the sense of distributions.

Existence result. Our main results are collected in the following theorems:

Theorem 2.3. Assume that (2.1)-(2.8) hold true. Then the problem (3.2) has at least one weak solution $u$.

Proof. The proof of theorem 2.3 is done in five steps. 
Step 1: Approximate problem and a priori estimates. For $n>0$, let us define the following approximation of $g, H$ and,$f$. First, set

$$
g_{n}(x, s, \xi)=\frac{g(x, s, \xi)}{1+\frac{1}{n}|g(x, s, \xi)|} \text { and } H_{n}(x, \xi)=\frac{H(x, \xi)}{1+\frac{1}{n}|H(x, \xi)|} .
$$

Note that $g_{n}(x, s, \xi)$ and $H_{n}(x, \xi)$ are satisfying the following conditions

$$
\left|g_{n}(x, s, \xi)\right| \leq n \quad \text { and } \quad\left|H_{n}(x, \xi)\right| \leq n .
$$

Let $f_{n}$ is a regular functions such that $f_{n}$ strongly converges to $f$ in $L^{1}(\Omega)$ and $\left\|f_{n}\right\|_{L^{1}} \leq c_{1}$ for some constant $c_{1}$.

Let us now consider the approximate problem

$$
-\operatorname{div}\left(a\left(x, u_{n}, \nabla u_{n}\right)\right)+g_{n}\left(x, u_{n}, \nabla u_{n}\right)+H_{n}\left(x, \nabla u_{n}\right)=f_{n}-\operatorname{div} F \text { in } \Omega .
$$

From the Leray-Lions existence theorem (cf. Theorem 2.1 and Remark 2.1 in chapter 2 of [22]), there exists at least one weak solution $u_{n} \in W_{0}^{1, p}(\Omega)$ of the approximate problem (2.11).

Now, we prove the solution $u_{n}$ of problem $(2.11)$ is bounded in $W_{0}^{1, p}(\Omega)$, we prove the following

Lemma 2.4. Let $u_{n} \in W_{0}^{1, p}(\Omega)$ be a weak solution of (2.11). Then, the following estimates holds,

$$
\left\|u_{n}\right\|_{W_{0}^{1, p}(\Omega)} \leq D
$$

where $D$ depend only on $\Omega, N, p, p^{\prime}, f, F$ and $\|b\|_{L^{r}(\Omega)}$.

Proof. To get (2.12), we divide the integral $\int_{\Omega}\left|\nabla u_{n}\right|^{p} d x$ in two parts and we prove the following estimates: for all $k \geq 0$

$$
\int_{\left\{\left|u_{n}\right| \leq k\right\}}\left|\nabla u_{n}\right|^{p} d x \leq M_{1} k
$$

and

$$
\int_{\left\{\left|u_{n}\right|>k\right\}}\left|\nabla u_{n}\right|^{p} d x \leq M_{2}
$$

where $M_{1}$ and $M_{2}$ are positive constants. In what follows we will denote by $M_{i}$, $i=3,4, \cdots$, some generic positive constants. For $\varepsilon>0$ and $s \geq 0$, we define

$$
\varphi_{\varepsilon}(r)= \begin{cases}\operatorname{sign}(r) & \text { if } \quad|r|>s+\varepsilon \\ \frac{\operatorname{sign}(r)(|r|-s)}{\varepsilon} & \text { if } s<|r| \leq s+\varepsilon \\ 0 & \text { otherwise. }\end{cases}
$$


We choose $v=\varphi_{\varepsilon}\left(u_{n}\right)$ as test function in (2.11), we have

$$
\begin{aligned}
& \int_{\Omega} a\left(x, u_{n}, \nabla u_{n}\right) \nabla\left(\varphi_{\varepsilon}\left(u_{n}\right)\right) d x \\
& +\int_{\Omega} g_{n}\left(x, u_{n}, \nabla u_{n}\right) \varphi_{\varepsilon}\left(u_{n}\right) d x+\int_{\Omega} H_{n}\left(x, \nabla u_{n}\right) \varphi_{\varepsilon}\left(u_{n}\right) d x \\
& =\int_{\Omega} f_{n} \varphi_{\varepsilon}\left(u_{n}\right) d x+\int_{\Omega} F \nabla\left(\varphi_{\varepsilon}\left(u_{n}\right)\right) d x .
\end{aligned}
$$

Using $g_{n}\left(x, u_{n}, \nabla u_{n}\right) \varphi_{\varepsilon}\left(u_{n}\right) \geq 0,(2.7)$ and Hölder's inequality, we obtain

$$
\begin{aligned}
& \frac{1}{\varepsilon} \int_{\left\{s<\left|u_{n}\right| \leq s+\varepsilon\right\}} a\left(x, u_{n}, \nabla u_{n}\right) \nabla u_{n} d x \\
& \leq\left(\int_{\left\{s<\left|u_{n}\right| \leq s+\varepsilon\right\}}|F|^{p^{\prime}} d x\right)^{\frac{1}{p^{\prime}}}\left(\int_{\left\{s<\left|u_{n}\right| \leq s+\varepsilon\right\}}\left(\frac{\left|\nabla u_{n}\right|}{\varepsilon}\right)^{p} d x d t\right)^{\frac{1}{p}} \\
& \quad+\int_{\left\{s<\left|u_{n}\right|\right\}} b(x)\left|\nabla u_{n}\right|^{p-1} d x+\int_{\left\{s<\left|u_{n}\right|\right\}}\left|f_{n}\right| d x
\end{aligned}
$$

Observe that,

$$
\begin{aligned}
\int_{\left\{s<\left|u_{n}\right|\right\}} b(x) \mid \nabla & \left.u_{n}\right|^{p-1} d x \\
& \leq \int_{s}^{+\infty}\left(\frac{-d}{d \sigma} \int_{\left\{\sigma<\left|u_{n}\right|\right\}} b^{p} d x\right)^{\frac{1}{p}}\left(\frac{-d}{d \sigma} \int_{\left\{\sigma<\left|u_{n}\right|\right\}}\left|\nabla u_{n}\right|^{p} d x\right)^{\frac{1}{p^{\prime}}} d \sigma .
\end{aligned}
$$

Because,

$$
\begin{aligned}
& \int_{\left\{s<\left|u_{n}\right|\right\}} b(x)\left|\nabla u_{n}\right|^{p-1} d x=\int_{s}^{+\infty} \frac{-d}{d \sigma}\left(\int_{\left\{\sigma<\left|u_{n}\right|\right\}} b(x)\left|\nabla u_{n}\right|^{p-1} d x\right) d \sigma \\
&= \int_{s}^{+\infty} \lim _{\delta \rightarrow 0} \frac{1}{\delta}\left(\int_{\left\{\sigma<\left|u_{n}\right| \leq \sigma+\delta\right\}} b(x)\left|\nabla u_{n}\right|^{p-1} d x\right) d \sigma \\
& \leq \int_{s}^{+\infty} \lim _{\delta \rightarrow 0} \frac{1}{\delta}\left(\int_{\left\{\sigma<\left|u_{n}\right| \leq \sigma+\delta\right\}} b^{p} d x\right)^{\frac{1}{p}}\left(\int_{\left\{\sigma<\left|u_{n}\right| \leq \sigma+\delta\right\}}\left|\nabla u_{n}\right|^{p} d x\right)^{\frac{1}{p^{\prime}}} d \sigma \\
&= \int_{s}^{+\infty}\left(\lim _{\delta \rightarrow 0} \frac{1}{\delta} \int_{\left\{\sigma<\left|u_{n}\right| \leq \sigma+\delta\right\}} b^{p} d x d t\right)^{\frac{1}{p}}\left(\lim _{\delta \rightarrow 0} \frac{1}{\delta} \int_{\left\{\sigma<\left|u_{n}\right| \leq \sigma+\delta\right\}}\left|\nabla u_{n}\right|^{p} d x\right)^{\frac{1}{p^{\prime}}} d \sigma \\
&= \int_{s}^{+\infty}\left(\frac{-d}{d \sigma} \int_{\left\{\sigma<\left|u_{n}\right|\right\}} b^{p} d x\right)^{\frac{1}{p^{\prime}}} d \sigma \\
&
\end{aligned}
$$

By (2.3) and (2.15), we deduce that

$$
\begin{aligned}
& \frac{1}{\varepsilon} \int_{\left\{s<\left|u_{n}\right| \leq s+\varepsilon\right\}} \alpha\left|\nabla u_{n}\right|^{p} d x \leq \int_{\left\{s<\left|u_{n}\right|\right\}}\left|f_{n}\right| d x \\
& +\left(\frac{1}{\varepsilon} \int_{\left\{s<\left|u_{n}\right| \leq s+\varepsilon\right\}}|F|^{p^{\prime}} d x\right)^{\frac{1}{p^{\prime}}}\left(\frac{1}{\varepsilon} \int_{\left\{s<\left|u_{n}\right| \leq s+\varepsilon\right\}}\left|\nabla u_{n}\right|^{p} d x\right)^{\frac{1}{p}} \\
& \quad+\int_{s}^{+\infty}\left(\frac{-d}{d \sigma} \int_{\left\{\sigma<\left|u_{n}\right|\right\}} b^{p} d x\right)^{\frac{1}{p}}\left(\frac{-d}{d \sigma} \int_{\left\{\sigma<\left|u_{n}\right|\right\}}\left|\nabla u_{n}\right|^{p} d x\right)^{\frac{1}{p^{\prime}}} d \sigma .
\end{aligned}
$$


Letting $\varepsilon$ go to zero, we obtain

$$
\begin{aligned}
& \frac{-d}{d s} \int_{\left\{s<\left|u_{n}\right|\right\}} \alpha\left|\nabla u_{n}\right|^{p} d x \leq \int_{\left\{s<\left|u_{n}\right|\right\}}\left|f_{n}\right| d x \\
& +\left(\frac{-d}{d s} \int_{\left\{s<\left|u_{n}\right|\right\}}|F|^{p^{\prime}} d x\right)^{\frac{1}{p^{\prime}}}\left(\frac{-d}{d s} \int_{\left\{s<\left|u_{n}\right|\right\}}\left|\nabla u_{n}\right|^{p} d x\right)^{\frac{1}{p}} \\
& \quad+\int_{s}^{+\infty}\left(\frac{-d}{d \sigma} \int_{\left\{\sigma<\left|u_{n}\right|\right\}} b^{p} d x\right)^{\frac{1}{p}}\left(\frac{-d}{d \sigma} \int_{\left\{\sigma<\left|u_{n}\right|\right\}}\left|\nabla u_{n}\right|^{p} d x\right)^{\frac{1}{p^{\prime}}} d \sigma
\end{aligned}
$$

where $\left\{s<\left|u_{n}\right|\right\}$ denotes the set $\left\{(x) \in \Omega, s<\left|u_{n}(x)\right|\right\}$ and $\mu(s)$ stands for the distribution function of $u_{n}$, that is $\mu(s)=\left|\left\{(x) \in \Omega,\left|u_{n}(x)\right|<s\right\}\right|$ for all $s \geq 0$.

Now, we recall the following inequality (see for example [21]), we have for almost every $s>0$

$$
1 \leq\left(N C_{N}^{\frac{1}{N}}\right)^{-1}(\mu(s))^{\frac{1}{N}-1}\left(-\mu^{\prime}(s)\right)^{\frac{1}{p^{\prime}}}\left(-\frac{d}{d s} \int_{\left\{s<\left|u_{n}\right|\right\}}\left|\nabla u_{n}\right|^{p} d x\right)^{\frac{1}{p}} .
$$

Using (2.17), we have

$$
\begin{aligned}
& \frac{-d}{d s} \int_{\left\{s<\left|u_{n}\right|\right\}} \alpha\left|\nabla u_{n}\right|^{p} d x=\alpha\left(\frac{-d}{d s} \int_{\left\{s<\left|u_{n}\right|\right\}}\left|\nabla u_{n}\right|^{p} d x\right)^{\frac{1}{p^{\prime}}}\left(\frac{-d}{d s} \int_{\left\{s<\left|u_{n}\right|\right\}}\left|\nabla u_{n}\right|^{p} d x\right)^{\frac{1}{p}} \\
& \leq\left(\int_{\left\{s<\left|u_{n}\right|\right\}}\left|f_{n}\right| d x\right)\left(N C_{N}^{\frac{1}{N}}\right)^{-1}(\mu(s))^{\frac{1}{N}-1}\left(-\mu^{\prime}(s)\right)^{\frac{1}{p^{\prime}}}\left(-\frac{d}{d s} \int_{\left\{s<\left|u_{n}\right|\right\}}\left|\nabla u_{n}\right|^{p} d x\right)^{\frac{1}{p}} \\
&+\left(\frac{-d}{d s} \int_{\left\{s<\left|u_{n}\right|\right\}}|F|^{p^{\prime}} d x\right)^{\frac{1}{p^{\prime}}}\left(\frac{-d}{d s} \int_{\left\{s<\left|u_{n}\right|\right\}}\left|\nabla u_{n}\right|^{p} d x\right)^{\frac{1}{p}} \\
&+\left(N C_{N}^{\frac{1}{N}}\right)^{-1}(\mu(s))^{\frac{1}{N}-1}\left(-\mu^{\prime}(s)\right)^{\frac{1}{p^{\prime}}}\left(\frac{-d}{d s} \int_{\left\{s<\left|u_{n}\right|\right\}}\left|\nabla u_{n}\right|^{p} d x\right)^{\frac{1}{p}} \\
& \times \int_{s}^{+\infty}\left(\frac{-d}{d \sigma} \int_{\left\{\sigma<\left|u_{n}\right|\right\}} b^{p} d x\right)^{\frac{1}{p}}\left(\frac{-d}{d \sigma} \int_{\left\{\sigma<\left|u_{n}\right|\right\}}\left|\nabla u_{n}\right|^{p} d x\right)^{\frac{1}{p^{\prime}}} d \sigma,
\end{aligned}
$$

which implies that

$$
\begin{aligned}
& \alpha\left(\frac{-d}{d s} \int_{\left\{s<\left|u_{n}\right|\right\}}\left|\nabla u_{n}\right|^{p} d x\right)^{\frac{1}{p^{\prime}}} \\
& \leq\left(N C_{N}^{\frac{1}{N}}\right)^{-1}(\mu(s))^{\frac{1}{N}-1}\left(-\mu^{\prime}(s)\right)^{\frac{1}{p^{\prime}}}\left(\int_{\left\{s<\left|u_{n}\right|\right\}}\left|f_{n}\right| d x\right)+\left(\frac{-d}{d s} \int_{\left\{s<\left|u_{n}\right|\right\}}|F|^{p^{\prime}} d x\right)^{\frac{1}{p^{\prime}}} \\
& +\left(N C_{N}^{\frac{1}{N}}\right)^{-1}(\mu(s))^{\frac{1}{N}-1}\left(-\mu^{\prime}(s)\right)^{\frac{1}{p^{\prime}}} \int_{s}^{+\infty}\left(\frac{-d}{d \sigma} \int_{\left\{\sigma<\left|u_{n}\right|\right\}} b^{p} d x\right)^{\frac{1}{p}}\left(\frac{-d}{d \sigma} \int_{\left\{\sigma<\left|u_{n}\right|\right\}}\left|\nabla u_{n}\right|^{p} d x\right)^{\frac{1}{p^{\prime}}} d \sigma .
\end{aligned}
$$

Now, we consider three functions $B, F_{1}$ and $\psi$ (see Lemma 2.2 of [1]) defined by

$$
\begin{gathered}
\int_{\left\{s<\left|u_{n}\right|\right\}} b^{p}(x) d x=\int_{0}^{\mu(s)} B^{p}(\sigma) d \sigma . \\
\int_{\left\{s<\left|u_{n}\right|\right\}}|F|^{p^{\prime}} d x=\int_{0}^{\mu(s)} F_{1}^{p^{\prime}}(\sigma) d \sigma \text { and } \psi(s)=\int_{\left\{s<\left|u_{n}\right|\right\}}\left|f_{n}\right| d x .
\end{gathered}
$$


We have

$$
\begin{gathered}
\|B\|_{L^{p}(\Omega)} \leq\|h\|_{L^{p}(\Omega)} \\
\left\|F_{1}\right\|_{L^{p^{\prime}}(\Omega)} \leq\|F\|_{L^{p^{\prime}}(\Omega)} \text { and }|\psi(s)| \leq\left\|f_{n}\right\|_{L^{1}(\Omega)} .
\end{gathered}
$$

From (2.19), (2.20) and (2.21) becomes

$$
\begin{aligned}
& \alpha\left(\frac{-d}{d s} \int_{\left\{s<\left|u_{n}\right|\right\}}\left|\nabla u_{n}\right|^{p} d x\right)^{\frac{1}{p^{\prime}}} \\
& \leq F_{1}(\mu(s))\left(-\mu^{\prime}(s)\right)^{\frac{1}{p^{\prime}}}+\left(N C_{N}^{\frac{1}{N}}\right)^{-1}(\mu(s))^{\frac{1}{N}-1}\left(-\mu^{\prime}(s)\right)^{\frac{1}{p^{\prime}}} \psi(s) \\
& +\left(N C_{N}^{\frac{1}{N}}\right)^{-1}(\mu(s))^{\frac{1}{N}-1}\left(-\mu^{\prime}(s)\right)^{\frac{1}{p^{\prime}}} \int_{s}^{+\infty} B(\mu(\nu))\left(-\mu^{\prime}(\nu)\right)^{\frac{1}{p}}\left(-\frac{d}{d \nu} \int_{\left\{\nu<\left|u_{n}\right|\right\}}\left|\nabla u_{n}\right|^{p} d x\right)^{\frac{1}{p^{\prime}}} d \nu .
\end{aligned}
$$

From Lemma 1.1, we obtain

$$
\begin{aligned}
& \alpha\left(\frac{-d}{d s} \int_{\left\{s<\left|u_{n}\right|\right\}}\left|\nabla u_{n}\right|^{p} d x\right)^{\frac{1}{p^{\prime}}} \\
& \leq F_{1}(\mu(s))\left(-\mu^{\prime}(s)\right)^{\frac{1}{p^{\prime}}}+\left(N C_{N}^{\frac{1}{N}}\right)^{-1}(\mu(s))^{\frac{1}{N}-1}\left(-\mu^{\prime}(s)\right)^{\frac{1}{p^{\prime}}} \psi(s) \\
& +\left(N C_{N}^{\frac{1}{N}}\right)^{-1}(\mu(s))^{\frac{1}{N}-1}\left(-\mu^{\prime}(s)\right)^{\frac{1}{p^{\prime}}} \int_{s}^{+\infty}\left[F_{1}(\mu(\sigma))+\left(N C_{N}^{\frac{1}{N}}\right)^{-1}(\mu(\sigma))^{\frac{1}{N}-1} \psi(\sigma)\right] \\
& \left.\quad \times B(\mu(\sigma))\left(-\mu^{\prime}(\sigma)\right) \exp \left(\int_{s}^{\sigma}\left(N C_{N}^{\frac{1}{N}}\right)^{-1}\right) B(\mu(r))(\mu(r))^{\frac{1}{N}-1}\left(-\mu^{\prime}(r)\right) d r\right) d \sigma .
\end{aligned}
$$

Now, by a variable change and by Hölder inequality, we estimate the argument of the exponential function on the right hand side of (2.23)

$$
\begin{aligned}
\int_{s}^{\sigma} B(\mu(r))(\mu(r))^{\frac{1}{N}-1}\left(-\mu^{\prime}(r)\right) d r & =\int_{s}^{\sigma} B(z) z^{\frac{1}{N}-1} d z \\
& \leq \int_{0}^{|\Omega|} B(z) z^{\frac{1}{N}-1} d z \\
& \leq\|B\|_{L^{r}}\left(\int_{0}^{|\Omega|} z^{\left(\frac{1}{N}-1\right) r^{\prime}}\right)^{\frac{1}{r^{\prime}}}
\end{aligned}
$$

Raising to the power $p^{\prime}$ in (2.23) and we can write

$$
\frac{-d}{d s} \int_{\left\{s<\left|u_{n}\right|\right\}}\left|\nabla u_{n}\right|^{p} d x \leq M_{1} .
$$

where $M_{1}$ depend only on $\Omega, N, p, p^{\prime}, f, \alpha$ and $\|b\|_{L^{r}(\Omega)}$, integrating between 0 and $k$, and then (2.13) is proved.

We now give the proof of (2.14), using $T_{k}\left(u_{n}\right)$ as test function in (2.11), gives

$$
\begin{aligned}
\int_{\Omega} a(x, & \left.u_{n}, \nabla u_{n}\right) \nabla T_{k}\left(u_{n}\right) d x \\
& +\int_{\Omega}\left(g_{n}\left(x, u_{n}, \nabla u_{n}\right)+H_{n}\left(x, \nabla u_{n}\right)\right) T_{k}\left(u_{n}\right) d x \\
& =\int_{\Omega} f_{n} T_{k}\left(u_{n}\right) d x+\int_{\Omega} F \nabla T_{k}\left(u_{n}\right) d x .
\end{aligned}
$$


Using (2.7), we deduce that,

$$
\begin{aligned}
\int_{\left\{\left|u_{n}\right| \leq k\right\}} & a\left(x, u_{n}, \nabla u_{n}\right) \nabla u_{n} d x \\
& +\int_{\left\{\left|u_{n}\right| \leq k\right\}} g_{n}\left(x, u_{n}, \nabla u_{n}\right) u_{n} d x+\int_{\left\{\left|u_{n}\right|>k\right\}} g_{n}\left(x, u_{n}, \nabla u_{n}\right) T_{k}\left(u_{n}\right) d x \\
\leq & \int_{\Omega} f_{n} T_{k}\left(u_{n}\right) d x+\int_{\Omega} F \nabla T_{k}\left(u_{n}\right) d x+\int_{\Omega} b(x)\left|\nabla u_{n}\right|^{p-1}\left|T_{k}\left(u_{n}\right)\right| d x
\end{aligned}
$$

and by using in the fact that $g_{n}\left(x, u_{n}, \nabla u_{n}\right) u_{n} \geq 0$ and (2.3), we have

$$
\begin{aligned}
& \alpha \int_{\left\{\left|u_{n}\right| \leq k\right\}}\left|\nabla u_{n}\right|^{p} d x+\int_{\left\{\left|u_{n}\right|>k\right\}} g\left(x, u_{n}, \nabla u_{n}\right) T_{k}\left(u_{n}\right) d x \\
& \leq k|| f \|_{L^{1}}+\int_{\left\{\left|u_{n}\right| \leq k\right\}} F \nabla u_{n} d x+k \int_{\left\{\left|u_{n}\right| \leq k\right\}} b(x)\left|\nabla u_{n}\right|^{p-1} d x \\
& \quad+k \int_{\left\{\left|u_{n}\right| \geq k\right\}} b(x)\left|\nabla u_{n}\right|^{p-1} d x,
\end{aligned}
$$

which implies that,

$$
\begin{aligned}
& \int_{\left\{\left|u_{n}\right|>k\right\}} g\left(x, u_{n}, \nabla u_{n}\right) T_{k}\left(u_{n}\right) d x \\
& \leq k|| f \|_{L^{1}}+\int_{\left\{\left|u_{n}\right| \leq k\right\}} F \nabla u_{n} d x+k \int_{\left\{\left|u_{n}\right| \leq k\right\}} b(x)\left|\nabla u_{n}\right|^{p-1} d x \\
& \quad+k \int_{\left\{\left|u_{n}\right| \geq k\right\}} h(x)\left|\nabla u_{n}\right|^{p-1} d x .
\end{aligned}
$$

By Hölder inequality and (2.13), we obtain

$$
\begin{aligned}
& \int_{\left\{\left|u_{n}\right|>k\right\}} g\left(x, u_{n}, \nabla u_{n}\right) T_{k}\left(u_{n}\right) d x \\
& \leq k\|f\|_{L^{1}(\Omega)}+k M_{1}\|F\|_{\left(L^{p^{\prime}}(\Omega)\right)^{N}}+k^{1+\frac{1}{p^{\prime}}} M_{1}\|b\|_{L^{p}(\Omega)}+k \int_{\left\{\left|u_{n}\right|>k\right\}} b(x)\left|\nabla u_{n}\right|^{p-1} d x .
\end{aligned}
$$

From (2.6) and applying Young's inequality, we get for all $k>\delta$

$$
\begin{aligned}
\nu^{\prime} k \int_{\left\{\left|u_{n}\right|>k\right\}}\left|\nabla u_{n}\right|^{p} d x \leq & k\|f\|_{L^{1}(\Omega)}+k M_{1}|| F\left\|_{\left(L^{p^{\prime}}(\Omega)\right)^{N}}+k^{1+\frac{1}{p^{\prime}}} M_{1}\right\| b \|_{L^{p}(\Omega)} \\
& +k \int_{\left\{\left|u_{n}\right|>k\right\}} b(x)\left|\nabla u_{n}\right|^{p-1} d x \\
\leq & k\|f\|_{L^{1}(\Omega)}+k M_{1}|| F\left\|_{\left(L^{p^{\prime}}(\Omega)\right)^{N}}+k^{1+\frac{1}{p^{\prime}}} M_{1}\right\| b \|_{L^{p}(\Omega)} \\
& +M_{6} k \|\left. b\right|_{L^{p}} ^{p}+\frac{1}{p^{\prime}} \nu^{\prime} k \int_{\left\{\left|u_{n}\right|>k\right\}}\left|\nabla u_{n}\right|^{p} d x .
\end{aligned}
$$

Hence

$$
\begin{aligned}
& \left(1-\frac{1}{p^{\prime}}\right) \int_{\left\{\left|u_{n}\right|>k\right\}}\left|\nabla u_{n}\right|^{p} d x \\
& \quad \leq M_{3}\|f\|_{L^{1}(\Omega)}+M_{4}\|F\|_{\left(L^{p^{\prime}}(\Omega)\right)^{N}}+k^{\frac{1}{p^{\prime}}} M_{5}\|b\|_{L^{p}(\Omega)}+M_{7}\|b\|_{L^{p}}^{p},
\end{aligned}
$$


and Lemma 2.4 is proved.

Step 2: Almost everywhere convergence of $u_{n}$. We prove that $u_{n}$ converges to some function $u$ locally in measure (and therefore, we can always assume that the convergence is a.e. after passing to a suitable subsequence). We will show that $u_{n}$ is a Cauchy sequence in measure in any ball $B_{R}$.

Let $k>0$ large enough, we have

$$
\begin{aligned}
k \operatorname{meas}\left(\left\{\left|u_{n}\right|>k\right\} \cap B_{R}\right) & =\int_{\left\{\left|u_{n}\right|>k\right\} \cap B_{R}}\left|T_{k}\left(u_{n}\right)\right| d x \\
& \leq \int_{B_{R}}\left|T_{k}\left(u_{n}\right)\right| d x \\
& \leq C\left(\int_{\Omega}\left|\nabla T_{k}\left(u_{n}\right)\right|^{p} d x\right)^{\frac{1}{p}} \\
& \leq c_{1},
\end{aligned}
$$

which implies

$$
\operatorname{meas}\left(\left\{\left|u_{n}\right|>k\right\} \cap B_{R}\right) \leq \frac{c_{1}}{k^{1}}, \text { for all } k>1 .
$$

We have, for every $\delta>0$,

$$
\begin{aligned}
& \operatorname{meas}\left(\left\{\left|u_{m}-u_{n}\right|>\delta\right\} \cap B_{R}\right) \leq \operatorname{meas}\left(\left\{\left|u_{n}\right|>k\right\} \cap B_{R}\right) \\
& \quad+\operatorname{meas}\left(\left\{\left|u_{m}\right|>k\right\} \cap B_{R}\right)+\operatorname{meas}\left(\left\{\left|T_{k}\left(u_{n}\right)-T_{k}\left(u_{m}\right)\right|>\delta\right\}\right) .
\end{aligned}
$$

Since $T_{k}\left(u_{n}\right)$ is bounded in $W_{0}^{1, p}(\Omega)$, there exists some $v_{k} \in W_{0}^{1, p}(\Omega)$, such that

$$
\begin{aligned}
& T_{k}\left(u_{n}\right) \rightarrow v_{k} \text { weakly in } W_{0}^{1, p}(\Omega), \\
& T_{k}\left(u_{n}\right) \rightarrow v_{k} \text { strongly in } L^{p}(\Omega) \text { and a.e. in } \Omega .
\end{aligned}
$$

Consequently, we can assume that $T_{k}\left(u_{n}\right)$ is a Cauchy sequence in measure in $\Omega$.

Let $\varepsilon>0$, then, by (2.31) and (2.32), there exists some $k(\varepsilon)>0$ such that $\operatorname{meas}\left(\left\{\left|u_{n}-u_{m}\right|>\delta\right\} \cap B_{R}\right)<\varepsilon$ for all $n, m \geq n_{0}(k(\varepsilon), \delta, R)$. This proves that $\left(u_{n}\right)$ is a Cauchy sequence in measure in $B_{R}$, thus converges almost everywhere to some measurable function $u$. Then

$$
\begin{aligned}
& T_{k}\left(u_{n}\right) \rightarrow T_{k}(u) \text { weakly in } W_{0}^{1, p}(\Omega), \\
& T_{k}\left(u_{n}\right) \rightarrow T_{k}(u) \text { strongly in } L^{p}(\Omega) \text { and a.e. in } \Omega,
\end{aligned}
$$

which implies, by using (2.1), for all $k>0$ there exists a function $h_{k} \in\left(L^{p^{\prime}}(\Omega)\right)^{N}$, such that

$$
a\left(x, T_{k}\left(u_{n}\right), \nabla T_{k}\left(u_{n}\right)\right) \rightarrow h_{k} \text { weakly in }\left(L^{p^{\prime}}(\Omega)\right)^{N} .
$$

Step 3: Strong convergence of truncations. Let $k>0$, we consider the function $\phi(s)=s e^{\lambda s^{2}}$, with $\lambda \geq\left(\frac{L_{1}(k)}{\alpha}\right)^{2}$, we have the following inequality

$$
\phi^{\prime}(s)-\frac{L_{1}(k)}{\alpha}|\phi(s)| \geq \frac{1}{2}
$$

holds for all $s \in \mathbb{R}$. Here, we define $w_{n}=T_{2 k}\left(u_{n}-T_{h}\left(u_{n}\right)+T_{k}\left(u_{n}\right)-T_{k}(u)\right)$ where $h>2 k>0$, and the following function

$$
v_{n}=\phi\left(w_{n}\right)
$$


The use of $v_{n}$ as test function in (2.11), we obtain

$$
\begin{aligned}
\int_{\Omega} a(x, & \left.u_{n}, \nabla u_{n}\right) \cdot \nabla w_{n} \phi^{\prime}\left(w_{n}\right) d x+\int_{\Omega}\left(g_{n}\left(x, u_{n}, \nabla u_{n}\right)+H_{n}\left(x, \nabla u_{n}\right)\right) \phi\left(w_{n}\right) d x \\
= & \int_{\Omega} f_{n} \phi\left(w_{n}\right) d x+\int_{\Omega} F \cdot \nabla \phi\left(w_{n}\right) d x .
\end{aligned}
$$

Note that, $\nabla w_{n}=0$ on the set where $\left\{\left|u_{n}\right|>h+4 k\right\}$, therefore, setting $M=$ $4 k+h$, and denoting by $\alpha_{h}^{1}(n), \alpha_{h}^{2}(n), \cdots$, various sequences of real numbers which converge to zero when $n$ tends to infinity for any fixed value of $h$, we get by $(2.36)$

$$
\begin{aligned}
\int_{\Omega} a(x, & \left.T_{M}\left(u_{n}\right), \nabla T_{M}\left(u_{n}\right)\right) \cdot \nabla w_{n} \phi^{\prime}\left(w_{n}\right) d x+\int_{\Omega} g_{n}\left(x, u_{n}, \nabla u_{n}\right) \phi\left(w_{n}\right) d x \\
\leq & \int_{\Omega} f_{n} \phi\left(w_{n}\right) d x+\int_{\Omega} F \cdot \nabla \phi\left(w_{n}\right) d x+\int_{\Omega}\left|H_{n}\left(x, \nabla u_{n}\right) \phi\left(w_{n}\right)\right| d x .
\end{aligned}
$$

Since

$$
\left|\int_{\Omega} H_{n}\left(x, \nabla u_{n}\right) \phi\left(w_{n}\right) d x\right| \leq\left\|\nabla u_{n}\right\|_{L^{p}(\Omega)}^{p-1}\left\|b \phi\left(T_{2 k}\left(u-T_{h}(u)\right)\right)\right\|_{L^{p}}
$$

(where $b \phi\left(w_{n}\right) \rightarrow b \phi\left(T_{2 k}\left(u-T_{h}(u)\right)\right)$ in $L^{p}$, by Lebesque's dominated convergence theorem, because $\phi\left(w_{n}\right)$ is bounded ).

$$
\left|\int_{\Omega} H_{n}\left(x, \nabla u_{n}\right) \phi\left(w_{n}\right) d x\right|=M_{9}|| b \phi\left(T_{2 k}\left(u-T_{h}(u)\right)\right) \|_{L^{p}}+\alpha_{h}^{3}(n),
$$

and since $g_{n}\left(x, u_{n}, \nabla u_{n}\right) \phi\left(w_{n}\right) \geq 0$ on the subset $\left\{x \in \Omega:\left|u_{n}(x)\right|>k\right\}$, we deduce from (2.37) that

$$
\begin{aligned}
\int_{\Omega} a(x, & \left.T_{M}\left(u_{n}\right), \nabla T_{M}\left(u_{n}\right)\right) \cdot \nabla w_{n} \phi^{\prime}\left(w_{n}\right) d x+\int_{\left\{\left|u_{n}(x)\right| \leq k\right\}} g_{n}\left(x, u_{n}, \nabla u_{n}\right) \phi\left(w_{n}\right) d x \\
\leq & \int_{\Omega} f_{n} \phi\left(w_{n}\right) d x+\int_{\Omega} F \cdot \nabla \phi\left(w_{n}\right) d x+M_{9}|| b \phi\left(T_{2 k}\left(u-T_{h}(u)\right)\right) \|_{L^{p}}+\alpha_{h}^{3}(n) .
\end{aligned}
$$

Splitting the first integral on the left hand side of (2.40) where $\left|u_{n}\right| \leq k$ and $\left|u_{n}\right|>k$, we can write, by using (2.3)

$$
\begin{aligned}
& \int_{\Omega} a\left(x, T_{M}\left(u_{n}\right), \nabla T_{M}\left(u_{n}\right)\right) \cdot \nabla w_{n} \phi^{\prime}\left(w_{n}\right) d x \\
\geq & \int_{\Omega} a\left(x, T_{k}\left(u_{n}\right), \nabla T_{k}\left(u_{n}\right)\right) \cdot\left(\nabla T_{k}\left(u_{n}\right)-\nabla T_{k}(u)\right) \phi^{\prime}\left(w_{n}\right) d x \\
- & C_{k} \int_{\left\{\left|u_{n}\right|>k\right\}}\left|a\left(x, T_{M}\left(u_{n}\right), \nabla T_{M}\left(u_{n}\right)\right)\right|\left|\nabla T_{k}\left(u_{n}\right)\right| d x .
\end{aligned}
$$

where $C_{k}=\phi^{\prime}(2 k)$. Since, when $n$ tends to infinity, we have $\nabla T_{k}(u) \chi_{\left\{\left|u_{n}\right|>k\right\}}$ tends to 0 strongly in $\left(L^{p}(\Omega)\right)^{N}$ while, $\left(a\left(x, T_{M}\left(u_{n}\right), \nabla T_{M}\left(u_{n}\right)\right)\right)_{n}$ is bounded in $\left(L^{p^{\prime}}(\Omega)\right)^{N}$ hence the last term in the previous inequality tends to zero for every 
$h$ fixed as $n$ tends to infinity. Now, observe that

$$
\begin{aligned}
& \int_{\Omega} a\left(x, T_{k}\left(u_{n}\right), \nabla T_{k}\left(u_{n}\right)\right) \cdot\left[\nabla T_{k}\left(u_{n}\right)-\nabla T_{k}(u)\right] \phi^{\prime}\left(w_{n}\right) d x \\
& =\int_{\Omega}\left[a\left(x, T_{k}\left(u_{n}\right), \nabla T_{k}\left(u_{n}\right)\right)-a\left(x, T_{k}\left(u_{n}\right), \nabla T_{k}(u)\right)\right] \\
& \cdot\left[\nabla T_{k}\left(u_{n}\right)-\nabla T_{k}(u)\right] \phi^{\prime}\left(w_{n}\right) d x \\
& +\int_{\Omega} a\left(x, T_{k}\left(u_{n}\right), \nabla T_{k}(u)\right) \cdot\left[\nabla T_{k}\left(u_{n}\right)-\nabla T_{k}(u)\right] \phi^{\prime}\left(w_{n}\right) d x .
\end{aligned}
$$

By the continuity of the Nymetskii operator, we have for all $i=1, \cdots, N$.

$$
a_{i}\left(x, T_{k}\left(u_{n}\right), \nabla T_{k}(u)\right) \phi^{\prime}\left(w_{n}\right) \rightarrow a_{i}\left(x, T_{k}(u), \nabla T_{k}(u)\right) \phi^{\prime}\left(T_{2 k}\left(u-T_{k}(u)\right)\right)
$$

strongly in $L^{p^{\prime}}(\Omega)$, and since $\frac{\partial\left(T_{k}\left(u_{n}\right)\right)}{\partial x_{i}} \rightarrow \frac{\partial\left(T_{k}(u)\right)}{\partial x_{i}}$ weakly in $L^{p}(\Omega)$, the second term of the right hand side of (2.42) tends to zero as $n$ tends to infinity. So that (2.41) yields

$$
\begin{gathered}
\int_{\Omega} a\left(x, T_{M}\left(u_{n}\right), \nabla T_{M}(u)\right) \cdot\left[\nabla T_{k}\left(u_{n}\right)-\nabla T_{k}(u)\right] \phi^{\prime}\left(w_{n}\right) d x \\
\geq \int_{\Omega}\left[a\left(x, T_{k}\left(u_{n}\right), \nabla T_{k}\left(u_{n}\right)\right)-a\left(x, T_{k}\left(u_{n}\right), \nabla T_{k}(u)\right)\right] \\
\cdot\left[\nabla T_{k}\left(u_{n}\right)-\nabla T_{k}(u)\right] \phi^{\prime}\left(w_{n}\right) d x+\alpha_{h}^{5}(n) .
\end{gathered}
$$

For the second term of the left hand side of (2.40), we can estimate as follows

$$
\begin{aligned}
& \int_{\left\{\left|u_{n}\right| \leq k\right\}} g\left(x, u_{n}, \nabla u_{n}\right) \phi\left(w_{n}\right) d x \\
& \leq \int_{\left\{\left|u_{n}\right| \leq k\right\}} L_{1}(k)\left(L_{2}(x)+\left|\nabla T_{k}\left(u_{n}\right)\right|^{p}\right)\left|\phi\left(w_{n}\right)\right| d x \\
& \leq L_{1}(k) \int_{\Omega} L_{2}(x)\left|\phi\left(w_{n}\right)\right| d x \\
& \quad+\frac{L_{1}(k)}{\alpha} \int_{\Omega} a\left(x, T_{k}\left(u_{n}\right), \nabla T_{k}\left(u_{n}\right)\right) \cdot \nabla T_{k}\left(u_{n}\right)\left|\phi\left(w_{n}\right)\right| d x .
\end{aligned}
$$

Note that, we have

$$
\begin{aligned}
& \int_{\Omega} a\left(x, T_{k}\left(u_{n}\right), \nabla T_{k}\left(u_{n}\right)\right) \cdot \nabla T_{k}\left(u_{n}\right)\left|\phi\left(w_{n}\right)\right| d x \\
= & \int_{\Omega}\left[a\left(x, T_{k}\left(u_{n}\right), \nabla T_{k}\left(u_{n}\right)\right)-a\left(x, T_{k}\left(u_{n}\right), \nabla T_{k}(u)\right)\right] \cdot\left[\nabla T_{k}\left(u_{n}\right)-\nabla T_{k}(u)\right]\left|\phi\left(w_{n}\right)\right| d x \\
& +\int_{\Omega} a\left(x, T_{k}\left(u_{n}\right), \nabla T_{k}\left(u_{n}\right)\right) \cdot \nabla T_{k}(u)\left|\phi\left(w_{n}\right)\right| d x \\
& +\int_{\Omega} a\left(x, T_{k}\left(u_{n}\right), \nabla T_{k}(u)\right) \cdot\left[\nabla T_{k}\left(u_{n}\right)-\nabla T_{k}(u)\right]\left|\phi\left(w_{n}\right)\right| d x .
\end{aligned}
$$

By Lebesgue's Theorem, we have

$$
\nabla T_{k}(u)\left|\phi\left(w_{n}\right)\right| \rightarrow \nabla T_{k}(u)\left|\phi\left(T_{2 k}\left(u-T_{h}(u)\right)\right)\right| \text { strongly in }\left(L^{p}(\Omega)\right)^{N}
$$


Moreover, in view of (2.33) the second term of the right hand side of (2.45) tends to

$$
\int_{\Omega} h_{k} \cdot \nabla T_{k}(u)\left|\phi\left(T_{2 k}\left(u-T_{h}(u)\right)\right)\right| d x
$$

The third term of the right hand side of (2.45) tends to 0 since for all $i=1, \cdots, N$.

$$
a_{i}\left(x, T_{k}\left(u_{n}\right), \nabla T_{k}(u)\right) \phi\left(w_{n}\right) \rightarrow a_{i}\left(x, T_{k}(u), \nabla T_{k}(u)\right) \phi\left(T_{2 k}\left(u-T_{k}(u)\right)\right)
$$

strongly in $L^{p^{\prime}}(\Omega)$, while

$$
\frac{\partial\left(T_{k}\left(u_{n}\right)\right)}{\partial x_{i}} \rightarrow \frac{\partial\left(T_{k}(u)\right)}{\partial x_{i}} \text { weakly in } L^{p}(\Omega)
$$

From (2.44) and (2.45), we obtain

$$
\begin{aligned}
\left|\int_{\left\{\left|u_{n}\right| \leq k\right\}} g\left(x, u_{n}, \nabla u_{n}\right) \phi\left(w_{n}\right) d x\right| \\
\leq \int_{\Omega}\left[a\left(x, T_{k}\left(u_{n}\right), \nabla T_{k}\left(u_{n}\right)\right)-a\left(x, T_{k}\left(u_{n}\right), \nabla T_{k}(u)\right)\right] \\
\cdot\left[\nabla T_{k}\left(u_{n}\right)-\nabla T_{k}(u)\right]\left|\phi\left(w_{n}\right)\right| d x \\
+\int_{\Omega} h_{k} \cdot \nabla T_{k}(u)\left|\phi\left(T_{2 k}\left(u-T_{h}(u)\right)\right)\right| d x \\
+L_{1}(k) \int_{\Omega} L_{2}(x)\left|\phi\left(w_{n}\right)\right| d x+\alpha_{h}^{10}(n),
\end{aligned}
$$

Now, by the strongly convergence of $f_{n}$ and the fact that

$$
w_{n} \rightarrow T_{2 k}\left(u-T_{k}(u)\right) \text { weakly in } W_{0}^{1, p}(\Omega) \text { and weakly } \text { in } L^{\infty}(\Omega) \text {, }
$$

and by combining (2.43) and (2.46), we conclude that

$$
\begin{aligned}
& \int_{\Omega}\left[a\left(x, T_{k}\left(u_{n}\right), \nabla T_{k}\left(u_{n}\right)\right)-a\left(x, T_{k}\left(u_{n}\right), \nabla T_{k}(u)\right)\right] \\
& \quad \cdot\left[\nabla T_{k}\left(u_{n}\right)-\nabla T_{k}(u)\right]\left(\phi^{\prime}\left(w_{n}\right)-\frac{L_{1}(k)}{\alpha}\left|\phi\left(w_{n}\right)\right|\right) d x \\
& \leq L_{1}(k) \int_{\Omega} L_{2}(x)\left|\phi\left(T_{2 k}\left(u-T_{h}(u)\right)\right)\right| d x+\int_{\Omega} f \phi\left(T_{2 k}\left(u-T_{h}(u)\right)\right) \mid d x \\
& \quad+\int_{\Omega} F \cdot \nabla T_{2 k}\left(u-T_{h}(u)\right) \phi^{\prime}\left(T_{2 k}\left(u-T_{h}(u)\right)\right) \mid d x \\
& \quad+\int_{\Omega} h_{k} \cdot \nabla T_{k}(u)\left|\phi\left(T_{2 k}\left(u-T_{h}(u)\right)\right)\right| d x+M_{9}|| b \phi\left(T_{2 k}\left(u-T_{h}(u)\right)\right) \|_{L^{p}}+\alpha_{h}^{11}(n),
\end{aligned}
$$


which and (2.34), implies that

$$
\begin{aligned}
& \int_{\Omega}\left[a\left(x, T_{k}\left(u_{n}\right), \nabla T_{k}\left(u_{n}\right)\right)-a\left(x, T_{k}\left(u_{n}\right), \nabla T_{k}(u)\right)\right] \\
& \quad \cdot\left[\nabla T_{k}\left(u_{n}\right)-\nabla T_{k}(u)\right] d x \\
& \leq 2 L_{1}(k) \int_{\Omega} L_{2}(x)\left|\phi\left(T_{2 k}\left(u-T_{h}(u)\right)\right)\right| d x+2 \int_{\Omega} f \phi\left(T_{2 k}\left(u-T_{h}(u)\right)\right) \mid d x \\
& +2 \int_{\Omega} F \cdot \nabla T_{2 k}\left(u-T_{h}(u)\right) \phi^{\prime}\left(T_{2 k}\left(u-T_{h}(u)\right)\right) \mid d x \\
& +2 \int_{\Omega} h_{k} \cdot \nabla T_{k}(u)\left|\phi\left(T_{2 k}\left(u-T_{h}(u)\right)\right)\right| d x+2 M_{9}\left\|b \phi\left(T_{2 k}\left(u-T_{h}(u)\right)\right)\right\|_{L^{p}}+\alpha_{h}^{12}(n),
\end{aligned}
$$

hence, passing to the limit over $n$, we obtain

$$
\begin{aligned}
\limsup _{n \rightarrow+\infty} \int_{\Omega} & {\left[a\left(x, T_{k}\left(u_{n}\right), \nabla T_{k}\left(u_{n}\right)\right)-a\left(x, T_{k}\left(u_{n}\right), \nabla T_{k}(u)\right)\right] } \\
& \cdot\left[\nabla T_{k}\left(u_{n}\right)-\nabla T_{k}(u)\right] d x \\
\leq & 2 L_{1}(k) \int_{\Omega} L_{2}(x)\left|\phi\left(T_{2 k}\left(u-T_{h}(u)\right)\right)\right| d x+2 \int_{\Omega} f \phi\left(T_{2 k}\left(u-T_{h}(u)\right)\right) \mid d x \\
& +2 M_{9}|| b \phi\left(T_{2 k}\left(u-T_{h}(u)\right)\right) \|_{L^{p}} \\
& 2 \int_{\Omega} h_{k} \cdot \nabla T_{k}(u)\left|\phi\left(T_{2 k}\left(u-T_{h}(u)\right)\right)\right| d x \\
& +2 \int_{\Omega} F \cdot \nabla T_{2 k}\left(u-T_{h}(u)\right) \phi^{\prime}\left(T_{2 k}\left(u-T_{h}(u)\right)\right) \mid d x+\alpha_{h}^{13}(n) .
\end{aligned}
$$

It remains to show, for our purposes, that the all terms on the right hand side of (2.50) converge to zero as $h$ goes to infinity. The only difficulty that exists is in the last term. For the other terms it suffices to apply Lebesgue's Theorem.

We deal with this term. Let us observe that, if we take $\phi\left(T_{2 k}\left(u_{n}-T_{h}\left(u_{n}\right)\right)\right)$ as test function in (2.11), use (2.3) and (2.7), we obtain

$$
\begin{aligned}
& \alpha \int_{\left\{h \leq\left|u_{n}\right| \leq h+2 k\right\}}\left|\nabla u_{n}\right|^{p} \phi^{\prime}\left(T_{2 k}\left(u_{n}-T_{h}\left(u_{n}\right)\right)\right) d x \\
& +\int_{\Omega} g_{n}\left(x, u_{n}, \nabla u_{n}\right) \phi\left(T_{2 k}\left(u_{n}-T_{h}\left(u_{n}\right)\right)\right) d x \\
& \leq \int_{\Omega} f_{n} \phi\left(T_{2 k}\left(u_{n}-T_{h}\left(u_{n}\right)\right)\right) d x+\int_{\left\{h \leq\left|u_{n}\right| \leq h+2 k\right\}} F \cdot \nabla u_{n} \phi^{\prime}\left(T_{2 k}\left(u_{n}-T_{h}\left(u_{n}\right)\right)\right) d x \\
& +\int_{\Omega} b(x)\left|\nabla u_{n}\right|^{p-1}\left|\phi\left(T_{2 k}\left(u_{n}-T_{h}\left(u_{n}\right)\right)\right)\right| d x
\end{aligned}
$$


thanks to the sign condition (2.5), (2.6) and Young inequality, we get

$$
\begin{gathered}
\alpha \int_{\left\{h \leq\left|u_{n}\right| \leq h+2 k\right\}}\left|\nabla u_{n}\right|^{p} \phi^{\prime}\left(T_{2 k}\left(u_{n}-T_{h}\left(u_{n}\right)\right)\right) d x \\
+\nu^{\prime} \int_{\Omega}\left|\nabla u_{n}\right|^{p}\left|\phi\left(T_{2 k}\left(u_{n}-T_{h}\left(u_{n}\right)\right)\right)\right| d x \\
\leq \int_{\Omega} f_{n} \phi\left(T_{2 k}\left(u_{n}-T_{h}\left(u_{n}\right)\right)\right) d x \\
+\int_{\left\{h \leq\left|u_{n}\right| \leq h+2 k\right\}} F \cdot \nabla u_{n} \phi^{\prime}\left(T_{2 k}\left(u_{n}-T_{h}\left(u_{n}\right)\right)\right) d x \\
+C\left(p, p^{\prime}, N, \nu^{\prime}\right) \int_{\Omega}|b(x)|^{p}\left|\phi\left(T_{2 k}\left(u_{n}-T_{h}\left(u_{n}\right)\right)\right)\right| d x \\
+\nu^{\prime} \int_{\Omega}\left|\nabla u_{n}\right|^{p}\left|\phi\left(T_{2 k}\left(u_{n}-T_{h}\left(u_{n}\right)\right)\right)\right| d x .
\end{gathered}
$$

Hence

$$
\begin{aligned}
\alpha \int_{\left\{h \leq\left|u_{n}\right| \leq h+2 k\right\}}\left|\nabla u_{n}\right|^{p} \phi^{\prime}\left(T_{2 k}\left(u_{n}-T_{h}\left(u_{n}\right)\right)\right) d x \\
\leq \int_{\Omega} f_{n} \phi\left(T_{2 k}\left(u_{n}-T_{h}\left(u_{n}\right)\right)\right) d x \\
\quad+\int_{\left\{h \leq\left|u_{n}\right| \leq h+2 k\right\}} F \cdot \nabla u_{n} \phi^{\prime}\left(T_{2 k}\left(u_{n}-T_{h}\left(u_{n}\right)\right)\right) d x \\
\quad+C\left(p, p^{\prime}, N, \nu^{\prime}\right) \int_{\Omega}|b(x)|^{p}\left|\phi\left(T_{2 k}\left(u_{n}-T_{h}\left(u_{n}\right)\right)\right)\right| d x .
\end{aligned}
$$

Using the Young inequality, we have

$$
\begin{aligned}
& \frac{\alpha}{2} \int_{\left\{h \leq\left|u_{n}\right| \leq h+2 k\right\}}\left|\nabla u_{n}\right|^{p} \phi^{\prime}\left(T_{2 k}\left(u_{n}-T_{h}\left(u_{n}\right)\right)\right) d x \\
& \leq \int_{\Omega} f_{n} \phi\left(T_{2 k}\left(u_{n}-T_{h}\left(u_{n}\right)\right)\right) d x+C_{k} \int_{\left\{h \leq\left|u_{n}\right|\right\}}|F|^{p^{\prime}} d x \\
& \quad+C\left(p, p^{\prime}, N, \nu^{\prime}\right) \int_{\Omega}|b(x)|^{p}\left|\phi\left(T_{2 k}\left(u_{n}-T_{h}\left(u_{n}\right)\right)\right)\right| d x
\end{aligned}
$$

so that, since $\phi^{\prime} \geq 1$, we have

$$
\int_{\Omega}\left|\nabla T_{2 k}\left(u-T_{h}(u)\right)\right|^{p} d x \leq \int_{\Omega}\left|\nabla T_{2 k}\left(u-T_{h}(u)\right)\right|^{p} \phi^{\prime}\left(T_{2 k}\left(u-T_{h}(u)\right)\right) d x
$$

again because the norm is lower semi-continuity, we get

$$
\begin{aligned}
\int_{\Omega} \mid \nabla T_{2 k} & \left.\left(u-T_{h}(u)\right)\right|^{p} \phi^{\prime}\left(T_{2 k}\left(u-T_{h}(u)\right)\right) d x \\
\leq & C_{k} \int_{\Omega}\left|\nabla T_{2 k}\left(u-T_{h}(u)\right)\right|^{p} d x \\
\leq & C_{k} \liminf _{n \rightarrow+\infty} \int_{\Omega}\left|\nabla T_{2 k}\left(u_{n}-T_{h}\left(u_{n}\right)\right)\right|^{p} d x \\
\leq & C_{k} \liminf _{n \rightarrow+\infty} \int_{\Omega}\left|\nabla T_{2 k}\left(u_{n}-T_{h}\left(u_{n}\right)\right)\right|^{p} d x \phi^{\prime}\left(T_{2 k}\left(u_{n}-T_{h}\left(u_{n}\right)\right)\right) .
\end{aligned}
$$


Consequently, in view of (2.54) and (2.56), we obtain

$$
\begin{aligned}
\int_{\Omega} \mid \nabla T_{2 k}(u- & \left.T_{h}(u)\right)\left.\right|^{p} \phi^{\prime}\left(T_{2 k}\left(u-T_{h}(u)\right)\right) d x \\
\leq C_{k} & \liminf _{n \rightarrow+\infty} \int_{\left\{h \leq\left|u_{n}\right|\right\}}|F|^{p^{\prime}} d x \\
& +\liminf _{n \rightarrow+\infty} \int_{\Omega} f_{n} \phi\left(T_{2 k}\left(u_{n}-T_{h}\left(u_{n}\right)\right)\right) d x \\
& +C\left(p, p^{\prime}, N, \nu^{\prime}\right) \liminf _{n \rightarrow+\infty} \int_{\Omega}|b(x)|^{p}\left|\phi\left(T_{2 k}\left(u_{n}-T_{h}\left(u_{n}\right)\right)\right)\right| d x
\end{aligned}
$$

Finally, the strong convergence in $L^{1}(\Omega)$ of $f_{n}$, and $b \in L^{r}(\Omega)$, we have, as first $n$ and then $h$ tend to infinity,

$$
\limsup _{h \rightarrow+\infty} \int_{\{h \leq|u| \leq h+2 k\}}|\nabla u|^{p} \phi^{\prime}\left(T_{2 k}\left(u-T_{h}(u)\right)\right) d x=0
$$

hence

$$
\limsup _{h \rightarrow+\infty} \int_{\Omega} F \cdot \nabla T_{2 k}\left(u-T_{h}(u)\right) \phi^{\prime}\left(T_{2 k}\left(u-T_{h}(u)\right)\right) d x=0
$$

Therefore by (2.50), letting $h$ go to infinity, we conclude,

$$
\begin{aligned}
& \lim _{n \rightarrow+\infty} \int_{\Omega}\left[a\left(x, T_{k}\left(u_{n}\right), \nabla T_{k}\left(u_{n}\right)\right)-a\left(x, T_{k}\left(u_{n}\right), \nabla T_{k}(u)\right)\right] \cdot\left[\nabla T_{k}\left(u_{n}\right)-\nabla T_{k}(u)\right] d x \\
& \quad=0 .
\end{aligned}
$$

Then, Lemma 5 of [14] implies,

$$
T_{k}\left(u_{n}\right) \rightarrow T_{k}(u) \text { strongly in } W_{0}^{1, p}(\Omega)
$$

Step 4: Equi-integrability of $H_{n}$ and $g_{n}$. We shall now prove that $H_{n}\left(x, \nabla u_{n}\right)$ converges to $H(x, \nabla u)$ and $g_{n}\left(x, u_{n}, \nabla u_{n}\right)$ converges to $g(x, u, \nabla u)$ strongly in $L^{1}(\Omega)$ by using Vitali's theorem. Since $H_{n}\left(x, \nabla u_{n}\right) \rightarrow H(x, \nabla u)$ a.e. $\Omega$ and $g_{n}\left(x, u_{n}, \nabla u_{n}\right) \rightarrow g(x, u, \nabla u)$ a.e. $\Omega$, thanks to (2.4) and (2.7), it suffices to prove that $H_{n}\left(x, \nabla u_{n}\right)$ and $g_{n}\left(x, u_{n}, \nabla u_{n}\right)$ are uniformly equi-integrable in $\Omega$. We will now prove that $H_{n}\left(x, \nabla u_{n}\right)$ is uniformly equi-integrable, we use Hölder's inequality and (2.12), we have for any measurable subset $E \subset \Omega$ :

$$
\begin{aligned}
\int_{E}\left|H_{n}\left(x, \nabla u_{n}\right)\right| d x & \leq\left(\int_{E} b^{p}(x) d x\right)^{\frac{1}{p}}\left(\int_{\Omega}\left|\nabla u_{n}\right|^{p}\right)^{\frac{1}{p^{\prime}}} \\
& \leq C\left(\int_{E} b^{p}(x) d x\right)^{\frac{1}{p}}
\end{aligned}
$$

which is small uniformly in $n$ when the measure of $E$ is small. 
To prove the uniform equi-integrability of $g_{n}\left(x, u_{n}, \nabla u_{n}\right)$. For any measurable subset $E \subset \Omega$ and $m \geq 0$,

$$
\begin{aligned}
\int_{E} & \left|g\left(x, u_{n}, \nabla u_{n}\right)\right| d x \\
= & \int_{E \cap\left\{\left|u_{n}\right| \leq m\right\}}\left|g\left(x, u_{n}, \nabla u_{n}\right)\right| d x+\int_{E \cap\left\{\left|u_{n}\right|>m\right\}}\left|g\left(x, u_{n}, \nabla u_{n}\right)\right| d x \\
\leq & L_{1}(m) \int_{E \cap\left\{\left|u_{n}\right| \leq m\right\}}\left(L_{2}(x)+\left|\nabla u_{n}\right|^{p}\right) d x+\int_{E \cap\left\{\left|u_{n}\right|>m\right\}}\left|g\left(x, u_{n}, \nabla u_{n}\right)\right| d x \\
\leq & L_{1}(m) \int_{E \cap\left\{\left|u_{n}\right| \leq m\right\}}\left(L_{2}(x)+\left|\nabla T_{m}\left(u_{n}\right)\right|^{p}\right) d x \\
& +\int_{E \cap\left\{\left|u_{n}\right|>m\right\}}\left|g\left(x, u_{n}, \nabla u_{n}\right)\right| d x \\
= & K_{1}+K_{2} .
\end{aligned}
$$

For fixed $m$, we get

$$
K_{1} \leq L_{1}(m) \int_{E}\left(L_{2}(x)+\left|\nabla T_{m}\left(u_{n}\right)\right|^{p}\right) d x
$$

which is thus small uniformly in $n$ for $m$ fixed when the measure of $E$ is small (recall that $T_{m}\left(u_{n}\right)$ tends to $T_{m}(u)$ strongly in $W_{0}^{1, p}(\Omega)$ ). We now discuss the behavior of the second integral of the right hand side of $(2.61)$, let $\psi_{m}$ be a function such that

$$
\begin{cases}\psi_{m}(s)=0 & \text { if } \quad|s| \leq m-1 \\ \psi_{m}(s)=\operatorname{sign}(s) & \text { if } \quad|s| \geq m \\ \psi_{m}^{\prime}(s)=1 & \text { if } \quad m-1<|s|<m\end{cases}
$$

We chooses for $m>1, \psi_{m}\left(u_{n}\right)$ as a test function in (2.11), we obtain

$$
\begin{aligned}
& \int_{\Omega} a\left(x, u_{n}, \nabla u_{n}\right) \nabla u_{n} \psi_{m}^{\prime}\left(u_{n}\right) d x \\
& +\int_{\Omega} g_{n}\left(x, u_{n}, \nabla u_{n}\right) \psi_{m}\left(u_{n}\right) d x+\int_{\Omega} H_{n}\left(x, \nabla u_{n}\right) \psi_{m}\left(u_{n}\right) d x \\
& \quad=\int_{\Omega} f_{n} \psi_{m}\left(u_{n}\right) d x+\int_{\Omega} F \nabla u_{n} \psi_{m}^{\prime}\left(u_{n}\right) d x .
\end{aligned}
$$

Using (2.3) and Hölder's inequality

$$
\begin{gathered}
\int_{\left\{m-1 \leq\left|u_{n}\right|\right\}}\left|g_{n}\left(x, u_{n}, \nabla u_{n}\right)\right| d x \leq \int_{E}\left|H_{n}\left(x, \nabla u_{n}\right)\right| d x \\
+\int_{\left\{m-1 \leq\left|u_{n}\right|\right\}}|f| d x+\|F\|_{L^{p^{\prime}}(\Omega)}\left(\int_{\left\{m-1 \leq\left|u_{n}\right| \leq m\right\}}\left|\nabla u_{n}\right|^{p} d x\right)^{\frac{1}{p}},
\end{gathered}
$$

and by (2.12), we have

$$
\lim _{m \rightarrow \infty} \sup _{n \in \mathbb{N}} \int_{\left\{\left|u_{n}\right|>m-1\right\}}\left|g_{n}\left(x, u_{n}, \nabla u_{n}\right)\right| d x=0 .
$$

Thus we proved that the second term of the right hand side of (2.61) is also small, uniformly in $n$ and in $E$ when $m$ is sufficiently large, which shows that 
$g_{n}\left(x, u_{n}, \nabla u_{n}\right)$ and $H_{n}\left(x, \nabla u_{n}\right)$ are uniformly equi-integrable in $\Omega$ as required, we conclude that

$$
\begin{array}{lll}
H_{n}\left(x, \nabla u_{n}\right) \rightarrow H(x, \nabla u) & \text { strongly in } & L^{1}(\Omega), \\
g_{n}\left(x, u_{n}, \nabla u_{n}\right) \rightarrow g(x, u, \nabla u) & \text { strongly in } & L^{1}(\Omega) .
\end{array}
$$

Step 5: Passing to the limit. We take $T_{k}\left(u_{n}-v\right)$ as test function in (2.11), with $v \in W_{0}^{1, p}(\Omega) \cap L^{\infty}(\Omega)$, we can write

$$
\begin{aligned}
\int_{\Omega} a(x, & \left.T_{k+\|v\|_{\infty}}\left(u_{n}\right), \nabla T_{k+\|v\|_{\infty}}\left(u_{n}\right)\right) \cdot \nabla T_{k}\left(u_{n}-v\right) d x \\
& +\int_{\Omega}\left(g\left(x, u_{n}, \nabla u_{n}\right)+H\left(x, \nabla u_{n}\right)\right) T_{k}\left(u_{n}-v\right) d x \\
= & \int_{\Omega} f_{n} T_{k}\left(u_{n}-v\right) d x+\int_{\Omega} F \cdot \nabla T_{k}\left(u_{n}-v\right) d x
\end{aligned}
$$

By Fatou's lemma and in fact that

$$
a\left(x, T_{k+\|v\|_{\infty}}\left(u_{n}\right), \nabla T_{k+\|v\|_{\infty}}\left(u_{n}\right)\right) \rightarrow a\left(x, T_{k+\|v\|_{\infty}}(u), \nabla T_{k+\|v\|_{\infty}}(u)\right)
$$

weakly in $\left(L^{p^{\prime}}(\Omega)\right)^{N}$. It is easily see that

$$
\begin{aligned}
& \int_{\Omega} a\left(x, T_{k+\|v\|_{\infty}}(u), \nabla T_{k+\|v\|_{\infty}}(u)\right) \cdot \nabla T_{k}(u-v) d x \\
& \quad \leq \liminf _{n \rightarrow \infty} \int_{\Omega} a\left(x, T_{k+\|v\|_{\infty}}\left(u_{n}\right), \nabla T_{k+\|v\|_{\infty}}\left(u_{n}\right)\right) \cdot \nabla T_{k}\left(u_{n}-v\right) d x .
\end{aligned}
$$

For the second term of the right hand side of (2.64), we have

$$
\int_{\Omega} F \cdot \nabla T_{k}\left(u_{n}-v\right) d x \rightarrow \int_{\Omega} F \cdot \nabla T_{k}(u-v) d x \quad \text { as } \quad n \rightarrow+\infty,
$$

since $\nabla T_{k}\left(u_{n}-v\right) \rightarrow \nabla T_{k}(u-v)$ weakly in $\left(L^{p}(\Omega)\right)^{N}$. On the other hand, we have

$$
\int_{\Omega} f_{n} T_{k}\left(u_{n}-v\right) d x \rightarrow \int_{\Omega} f T_{k}(u-v) d x \text { as } n \rightarrow+\infty .
$$

Tanks to (2.63) and (2.65)-(2.67), we can pass to the limit in (2.64), and we obtain that $u$ is a solution of the problem (3.2). This completes the proof of Theorem 2.3 .

Remark 2.5. The condition (2.4) can be replaced by the weaker one

$$
|g(x, s, \xi)| \leq L_{2}(x)+L_{1}(|s|)|\xi|^{p}
$$

where $L_{1}: \mathbb{R}^{+} \rightarrow \mathbb{R}^{+}$is a continuous increasing function and $L_{2}(x) \in L^{1}(\Omega)$.

Remark 2.6. Note that the solution given by Theorem 2.3 belongs to the energy space $W_{0}^{1, p}(\Omega)$. This better regularity is due the assumption (2.6). Indeed, if (2.6) is not satisfied we cannot expect that the solution belongs to (2.6). One can see a counterexample in [13]. 


\section{EXAMPLE}

Let us consider

$$
a(x, s, \xi)=|\xi|^{p-2} \xi, \quad g(x, s, \xi)=\frac{s^{2}+1}{s^{2}+3} \operatorname{sign}(s)|\xi|^{p}, \quad H(x, \xi)=h(x)|\xi|^{p-1},
$$

where $h(x) \in L^{r}(\Omega)$ with $r>\max (N, p)$, with $x \in \Omega$. It is easy to show that the $a(x, s, \xi)$ are Carathéodory functions satisfying the growth condition (2.1), the coercivity (2.3) and the monotonicity condition (2.2).

While the Carathéodory function $g(x, s, \xi)$ satisfies the condition (2.4) indeed

$$
|g(x, s, \xi)| \leq \frac{s^{2}+1}{s^{2}+3}|\xi|^{p} \equiv L_{1}(s)|\xi|^{p}
$$

where $L_{1}(s)=\frac{s^{2}+1}{s^{2}+3}$ is clearly bounded continuous increasing function in $\mathbb{R}^{+}$.

Note that $g(x, s, \xi)$ satisfying the sign condition (2.5) and the coercivity condition (2.6), and the function $H(x, \xi)$ satisfy the condition (2.7).

Finally, the hypotheses of Theorem 2.3 are satisfied. Therefore, for all $f \in$ $L^{1}(\Omega)$ and $F \in\left(L^{p^{\prime}}(\Omega)\right)^{N}$, the following problem

$$
\begin{cases}-\operatorname{div}\left(|\nabla u|^{p-2} \nabla u\right)+\frac{u^{2}+1}{u^{2}+3} \operatorname{sign}(u)|\xi|^{p}+h(x)|\nabla u|^{p-1}=f-\operatorname{div} F & \text { in } \Omega, \\ u=0 & \text { in } \partial \Omega,\end{cases}
$$

has at least one solution in the sense of Definition 2.9.

\section{REFERENCES}

1. A. Alvino and G. Trombetti, Sulle migliori costanti di maggiorazione per una classe di equazioni ellittiche degeneri, (Italian) Ricerche Mat. 27 (1978), 413-428.

2. E. Beckenbak and R. Beliman, Inequalities, Springer-Verlag, 1965.

3. A. Benkirane, Approximations de type Hedberg dans les espaces $W^{m} L \log L(\Omega)$ et applications. (French) [Hedberg-type approximations in the spaces $W^{m} L \log L(\Omega)$ and applications], Ann. Fac. Sci. Toulouse Math. (5) 11 (1990), no. 2, 67-78.

4. A. Benkirane and J.P. Gossez, An approximation theorem for higher order Orlicz-Sobolev spaces, Studia Math 92 (1989), 231-255.

5. A. Benkirane and A. Elmahi, An existence theorem form a strongly nonlinear problems in Orlicz space, Nonlinear Anal. 3 (1999), no. 6, 11-24.

6. A. Benkirane and A. Elmahi, Strongly nonlinear elliptic equations having natural growth terms and $L^{1}$ data, Nonlinear Anal. 39 (2000), no. 4, 403-411.

7. A. Benkirane, A. Elmahi and D. Meskine, An existence theorem for a class of elliptic problems in $L^{1}$, Appl. Math. (Warsaw) 29 (2002), no. 4, 439-457.

8. A. Youssfi, A. Benkirane and M. El Moumni, Bounded solutions of unilateral problems for strongly nonlinear equations in Orlicz spaces, Electron. J. Qual. Theory Differ. Equ. 2013, No. 21, 25pp.

9. A. Youssfi, A. Benkirane and M. El Moumni, Existence result for strongly nonlinear elliptic unilateral problems with $L_{1}$-data, Complex Var. Elliptic Equ. 59 (2014), no. 4, 447-461.

10. A. Bensoussan, L. Boccardo and F. Murat, On a non linear partial differential equation having natural growth terms and unbounded solution, Ann. Inst. Poincaré 5 (1988), 347-364.

11. L. Boccardo and T. Gallouët, Nonliner elliptic equations with right hand side measure, Comm. Partial Differential Equations 17 (1992), no. 3-4, 641-655.

12. L. Boccardo and T. Gallouët, Strongly nonlinear elliptic equation having natural growth terms and $L^{1}$ data, Nonlinear Anal. 19 (1992), 573-578. 
13. L. Boccardo,T. Gallouët and L. Orsina, Existence and nonexistence of solutions for some nonlinear elliptic equations, J. Anal. Math. 73 (1997), 203-223.

14. L. Boccardo, F. Murat and J.P. Puel, Existance of bounded solution for non linear elliptic unilateral problems, Annali Mat. Pura Appl. 152 (1988), 183-196.

15. H. Brezis and W. Strauss, Semilinear second-order elliptic equations in $L^{1}$, J. Math. Soc. Japan 25, (1973), no. 4, 565-590.

16. T. Del Vecchio, Nonlinear elliptic equations with measure data, Potential Anal. 4 1995), no. 2, 185-203.

17. T. Gallouët and R. Herbin, Existence of a solution to a coupled elliptic system, Appl. Math. Lett. 7 (1994), 49-55.

18. T. Goudon and M. Saad, On a Fokker-Planck equation arising in population dynamics, Rev. Mat. Complut. 11 (1998), 353-372.

19. R. Lewandowski, The mathematical analysis of the coupling of a turbulent kinetic energy equation to the Navier-Stokes equation with an eddy-viscosity, Nonlinear Anal. T.M.A 28 (1997), 393-417.

20. J.-L. Lions, Mathematical topics in fluid mechanics, incompressible models, Oxford Lecture Series in Math and its Applications 3 Clarendon Press, 1996.

21. G. H. Hardy, Littlewood and G. Polya, Inequalities, Cambrige University Press, Cambrige, 1964.

22. J.-L. Lions, Quelques méthodes de résolution des problème aux limites non lineaires, Dundo, Paris, 1969.

23. V.M. Monetti and L. Randazzo, Existence results for nonlinear elliptic equations with $p$ growth in the gradient, Ricerche Mat. 49 (2000), no. 1, 163-181.

24. V. Radulescu and M. Willem, Elliptic systems involving finite Radon measures, Differential Integral Equations 16 (2003), no. 2, 221-229.

25. A. Porretta, Existence for elliptic equations in $L^{1}$ having lower order terms with natural growth, Portugual. Math. 57 (2000), 179-190.

26. G. Talenti, Nonlinear elliptic equations, rearrangements of functions and Orlicz spaces, Ann .Mat. Pura Appl. 120 (1979), 159-184.

${ }^{1}$ Laboratory LiMAO

Faculté Poly-disciplinaire de Taza, University Sidi Mohamed Ben Abdellah, P.O. Box 1223 Taza Gare, Morocco.

E-mail address: akdimyoussef@yahoo.fr

${ }^{2}$ University Sidi Mohamed Ben Abdellah, Faculty of Sciences Dhar El Mahraz, Laboratory LAMA, Department of Mathematics, B.P 1796 Atlas Fez, Morocco.

E-mail address: abd.benkirane@gmail.com

E-mail address: mostafaelmoumni@gmail.com 